\title{
Development and validation of the diagnostic scale of traditional Chinese medicine syndrome elements for diabetic kidney disease
}

\author{
Min Jiang ${ }^{1}$, Xi-En Lou ${ }^{1}$, Xianhui Zhang ${ }^{2}$, Qian Nan ${ }^{1}$, Xue Gao ${ }^{3}$, Hongfang Liu ${ }^{2}$ \\ ${ }^{1}$ Department of endocrinology, Third Affiliated Hospital Affiliated to Beijing University of Chinese Medicine, Beijing, China; ${ }^{2}$ Department of \\ Endocrinology and Nephrology, Renal Research Institute of Beijing University of Chinese Medicine, Dongzhimen Hospital affiliated to Beijing \\ University of Chinese Medicine, Beijing, China; ${ }^{3}$ Department of School of Traditional Chinese Medicine, Beijing University of Chinese Medicine, \\ Beijing, China \\ Contributions: (I) Conception and design: H Liu, M Jiang; (II) Administrative support: None; (III) Provision of study materials or patients: M Jiang, \\ XE Lou, X Zhang, Q Nan, X Gao; (IV) Collection and assembly of data: M Jiang; (V) Data analysis and interpretation: M Jiang; (VI) Manuscript \\ writing: All authors; (VII) Final approval of manuscript: All authors. \\ Correspondence to: Hongfang Liu. Department of Endocrinology and Nephrology, Renal Research Institute of Beijing University of Chinese Medicine, \\ Dongzhimen Hospital Affiliated to Beijing University of Chinese Medicine, Haiyuncang Road No. 5, Beijing 100700, China. Email: lhfdoctor@126.com.
}

Background To construct a traditional Chinese medicine (TCM) syndrome elements diagnostic scale for diabetic kidney disease (DKD).

Methods: A total of 492 DKD patients were included in the study. TCM symptoms, signs and tongue manifestation information of the patients were collected, which constituted the items of the TCM syndrome elements diagnostic scale. Frequency dominance method was used to screen the core items. Cluster analysis and factor analysis method were used to identify the syndrome elements. Correlation coefficient and regression analysis were used to determine the syndrome elements. Regression coefficient was used to determine the scale items, and the diagnostic threshold was established by receiver operating characteristic curve. By using the above statistical methods , TCM syndrome elements diagnostic scale was constructed, and confirmed via diagnostic tests of 150 patients.

Results: There were 61 items of TCM diagnostic descriptions, and we kept the most useful 32 after filtering. After extracting the syndrome elements, a TCM syndrome elements diagnostic rating scale for DKD containing 9 syndrome elements was constructed, which were qi deficiency syndrome, blood deficiency syndrome, yin deficiency syndrome, yang deficiency syndrome, excessive heat syndrome, qi stagnation syndrome, damp heat syndrome, blood stasis syndrome and phlegm turbidity syndrome. A small-sample clinical validation test of the scale showed sensitivity of $78.8-100 \%$, specificity of $84.3-100 \%$, and accuracy of $82.7-100 \%$.

Conclusions: We constructed a TCM syndrome elements diagnostic rating scale for DKD, providing a basis for the standardized study of TCM syndromes.

Koywords: Diabetic kidney disease (DKD); syndrome elements; traditional Chinese medicine (TCM); diagnostic scale

Submitted Sep 28, 2021. Accepted for publication Nov 18, 2021.

doi: 10.21037/apm-21-3147

View this article at: https://dx.doi.org/10.21037/apm-21-3147

\section{Introduction}

Diabetic kidney disease (DKD) is a common and serious complication of diabetes mellitus (DM). According to the literature, the prevalence of DKD in patients with type 2
DM in China is $10-40 \%$ (1). Since 2015 DKD has replaced chronic glomerulonephritis as the leading cause of chronic kidney disease and end-stage renal disease (ESRD) (2-4). Traditional Chinese medicine (TCM) has shown obvious advantages in preventing and treating DKD in terms of 
improving symptoms, protecting the kidneys, and delaying the occurrence and progression of disease (5-10). TCM is effective in reducing urinary protein and delaying kidney damage (11). Diagnostic criteria for DKD in TCM is based on the course of disease, disease development and pathological viscera. The pathogenesis of DKD is excess in nature and deficiency in superficiality.

However, the diagnosis and treatment of DKD by TCM lacks consistent, standardized, and quantitative criteria, which has significantly affected the promotion of syndrome differentiation theories, formulae and treatments, restricting the utilization of TCM for DKD. Further research is needed to establish objective, unified and practical syndrome differentiation standards. Therefore, the aim of this research was to build up the diagnostic scale of TCM syndrome elements for DKD and validate it with a prospective small-sample trial.

We present the following article in accordance with the STARD reporting checklist (available at https://dx.doi. org/10.21037/apm-21-3147).

\section{Methods}

\section{Subjects}

\section{Source of cases}

492 studied cases were extracted from among the DKD patients who visited the departments of nephrology at 10 participating centers between March 2014 and December 2015. There was no restriction on sex. All procedures performed in this study involving human participants were in accordance with the Declaration of Helsinki (as revised in 2013). The study was approved by ethics board of Dongzhimen Hospital affiliated to Beijing University of Chinese Medicine (No. ECPJ-BDY-2013-47) and informed consent was taken from all the patients.

\section{Diagnostic criteria}

Based on the Mogensen diabetic nephropathy (DN) diagnostic criteria (stages II, III, and IV), and the "Guidelines for the Prevention and Treatment of Type 2 Diabetes in China (2013 Edition)" issued by the Chinese Medical Association (12), the following staging criteria were formulated.

Micro-albuminuria: 2 urinary tests positive for microalbumin (random urine albumin/creatinine ratio (ACR), $2.5-30 \mathrm{mg} / \mathrm{mmol}$ for men, $3.5-30 \mathrm{mg} / \mathrm{mmol}$ for women); or $20-200 \mathrm{mg} / \mathrm{L} / 24 \mathrm{~h}$ for quantitative urinary albumin assessment; or 30-300 $\mathrm{mg} / 24 \mathrm{~h}$ for urinary albumin excretion rate (UAER). Proteinuria: ACR $>30 \mathrm{mg} / \mathrm{mmol}$; or 24 h quantitative urinary albumin assessment $>200 \mathrm{mg} / \mathrm{L}$; or UAER $>300 \mathrm{mg} / 24 \mathrm{~h}$; glomerular filtration rate gradually decreased but $\geq 15 \mathrm{~mL} / \mathrm{min}$ (without dialysis).

\section{Study inclusion criteria}

(I) Age between 18 and 75 years, with signed informed consent; (II) DM history; (III) meeting the diagnostic/ staging criteria for micro-albuminuria or proteinuria; (IV) exclusion of non-DKD disease.

Renal biopsy was required in the following situations: DM history $<10$ years; persistent proteinuria but no retinopathy; rapid deterioration of renal function; hematuria or microscopic red cell casts.

\section{Study exclusion criteria}

(I) Diagnosed primary glomerular disease, drug-induced renal damage, auto-immune or hematologic disease; (II) history of acute and critical illness such as diabetic ketoacidosis, myocardial infarction, or cerebrovascular accident within past 6 months; (III) comorbid with severe underlying disease of respiratory, gastrointestinal, or hematologic systems; (IV) comorbid with grade I-IV congestive heart failure; (V) concurrent infection or mental disease; (VI) malignancy; (VII) pregnancy or breastfeeding.

\section{Quality control}

Standard operating procedures (SOP) for the clinical trials SOPs were formulated by the research team, comprising 19 quality control plans in the five aspects of the clinical trials (preparation, initiation, mid-term coordination, data management, and termination), which were implemented strictly.

\section{Standardized 4-level auditing system}

The first level audit was carried out by inspectors at each participating center; the second level audit, including authenticity, accuracy and completeness of the case tables, research progress, source data validation and subsequent audit report, was carried out by an external auditing company; the third-level audit was carried out by the project-hosting institution (Dongzhimen Hospital Affiliated to Beijing University of Chinese Medicine); and the fourthlevel audit was done by the project supervising institution (as for 3rd-level audit).

\section{Standardized completion of TCM syndrome observation table}

To ensure standardized completion of the TCM syndrome 
observation table (see Section "formulation of DKD TCM syndrome observation table"), training of research team members was given with a subsequent training and qualification inspection of each participating center. After the training of the participating center research participants, 6 typical medical records were selected for evaluation of TCM syndromes. If consistency was $<85 \%$, the training was repeated until consistency was $>85 \%$. The following conditions were excluded from the syndrome evaluation: acute complications of DM such as diabetic ketoacidosis, hyperosmolar hyperglycemia syndrome, hypoglycemia, complicated acute infectious diseases, surgery within 2 weeks, and a history of physical or mental trauma.

\section{Research methods}

\section{Formulation of DKD TCM syndrome observation table}

On the basis of a literature search (13), five rounds of expert consultation were conducted in the form of meetings and questionnaires by the research team to formulate a DKD TCM syndrome observation table, including 10 syndrome elements (qi deficiency, blood deficiency, yin deficiency, yang deficiency, stagnation of qi, blood stasis, excessive heat, damp, damp heat, phlegm), and 61 items of TCM symptoms. Except for the yellow and greasy tongue coating as a diagnostic item for damp heat syndrome, the other tongue and pulse conditions in the observation table were only recorded but not included in the statistical analysis due to their strong subjectivity.

\section{Case collection}

Case information was collected for the clinical questionnaire that had 4 parts: patients' basic information, anthropometric indicators, TCM syndrome observation table, and laboratory test indicators.

(I) Basic information: name, sex, age, occupation, ethnicity, family history, past history, smoking history, drinking history, medication status, diet, physical activity status, etc.; (II) anthropometric indicators: waist circumference, hip circumference, waist-to-hip ratio, height, weight, body mass index $\left(\mathrm{kg} / \mathrm{m}^{2}\right)$; (III) TCM syndrome observation table: including 61 TCM symptom items and used to determine 10 syndromes; (IV) laboratory test indicators: fasting plasma glucose, glycosylated hemoglobin (HbA1c), blood routine examination; liver function including total protein, albumin (ALB); kidney function, including serum creatinine (SCr), blood urea nitrogen, uric acid; ions including potassium $(\mathrm{K})$, calcium (Ca), phosphorus $(\mathrm{P})$; blood lipids: total cholesterol, triglycerides (TG), low-density lipoprotein cholesterol (LDL-C), highdensity lipoprotein cholesterol (HDL-C); routine urinalysis, urinary micro-albumin, urinary creatinine, urinary ACR, 24-h quantitative urinary protein.

\section{Data entry}

The research team cooperated with the School of Information Engineering, Beijing University of Science and Technology to build a website as the platform for entering, examination, inspection, management and extraction of data. After collecting the clinical information, the researchers entered and submitted the data via the data management platform.

\section{Scale formation, evaluation and validation}

Scores were assigned to each syndrome element diagnosis item and the diagnostic threshold was determined. The scale was evaluated by sensitivity and specificity. After the scale was formed, 150 patients who attended the participating centers between January 2014 and December 2015 and were eligible for study inclusion were selected for clinical validation of the scale.

\section{Statistical analysis}

SPSS17.0 was used for statistical analysis. Analysis of variance and $t$-test were used for continuous data, and $\chi^{2}$ test was used for categorical data. $\mathrm{P}<0.05$ indicated statistical difference and $\mathrm{P}<0.01$ significant statistical difference. Frequency statistics, hierarchical clustering analysis, factor analysis, binomial logistic regression, and Spearman correlation were used to examine the relationship between syndrome elements and symptoms.

\section{Results}

\section{Scale items}

\section{Items excluded by frequency}

According to the research conventions of scales, and considering the reality of clinical practice, items with a frequency of $<10 \%$ were excluded, which were: scant and light menses (non-menopausal women), susceptibility to colds, low and weak cough, premature ejaculation, spermatorrhea, pleural effusion, ascites, oliguria, petechiae or ecchymosis on the skin, nausea, and vomiting. 
Table 1 Correlation between syndrome elements and symptoms

\begin{tabular}{ll}
\hline Syndrome element & Symptoms \\
\hline Qi deficiency & $\begin{array}{l}\text { Qi deficiency and disinclination to speak, fatigue, shortness of breath, lassitude and pain of the loins and knees, } \\
\text { loose teeth, hair loss, loss of libido, tinnitus and deafness, abdominal distension after eating, lack of appetite, weak } \\
\text { defecation, spontaneous sweating }\end{array}$ \\
Blood deficiency & $\begin{array}{l}\text { Pale lips and fingernails, pale and sallow complexion, lusterless nails, numbness of hands and feet, insomnia and } \\
\text { dreaminess, forgetfulness, dizziness }\end{array}$ \\
Yin deficiency & $\begin{array}{l}\text { Dry stools, dizziness, dry eyes, aversion to heat and sweating or night sweating, dark urine, dysphoria with feverish } \\
\text { sensation in chest, palms and soles, dry throat, lassitude and pain of the loins and knees, tinnitus and deafness }\end{array}$ \\
Yang deficiency & $\begin{array}{l}\text { Loose stools, frequent urination, abdominal cold pain, edema of face and feet, cold limbs, frequent nocturia, } \\
\text { deficiency of libido }\end{array}$ \\
Excessive heat & Constipation and deep-colored urine, thirst and desire for cold drinks, polyphagia with tendency to hunger \\
Qi stagnation & distension in the side ribs, distension in the abdomen, irritability or depression and sighing \\
Blood stasis & $\begin{array}{l}\text { Squamous and dry skin, dark or dull complexion, dark purple lips, localized pain especially at night, dark purple } \\
\text { tongue, cyanosis tortuous and bruising sublingual vein }\end{array}$ \\
Damp heat & Heavy limbs, yellow and greasy tongue coating, dry mouth and bitter mouth \\
Phlegm turbidity & Abdominal distension, sticky mouth, itchy skin.
\end{tabular}

Core symptom items identified by frequency

Items with a frequency ratio $>40 \%$ were identified as core TCM symptoms of DKD, in descending frequency order: fatigue $(63.08 \%)$, loss of libido $(49.09 \%)$, dry throat (48.48\%), lassitude and pain of the loins and knees (46.45\%), loose teeth and loss of hair (45.23\%), qi deficiency and disinclination to speak (43.61\%), and frequent nocturia (42.19\%).

\section{Extracted syndrome elements}

Extracted syndrome elements by cluster analysis

Hierarchical clustering (Ward method, or square sum of deviations) was used to cluster 50 variables. After consulting with experts, the research team considered it would be more appropriate to cluster the 50 items into 17 categories. To determine the syndrome elements of disease nature (e.g., cold, heat, deficiency and excess) and disease location (e.g., liver, heart, spleen), 9 syndrome elements of qi deficiency, blood deficiency, yin deficiency, yang deficiency, qi stagnation, blood stasis, heat accumulation, damp heat, and phlegm turbidity were extracted.

\section{Extracted syndrome elements by factor analysis}

KMO (Kaiser-Meyer-Olkin) and Bartlett's test of sphericity were used for the fitness test of factor analysis. The factor extraction principle was an eigenvalue $>1$. A total of 50 variables were included, and 14 factors with a common factor eigenvalue $>1$ were retained. The retained factors could explain $61.282 \%$ of the cumulative variance of all information. Each of these factors contained $\geq 1$ symptoms.

A total of 9 syndrome elements-qi deficiency, blood deficiency, qi stagnation, yin deficiency, heat accumulation, yang deficiency, blood stasis, damp heat, and phlegm turbidity-were extracted through factor analysis. Based on professional knowledge, we made clinical judgments on the syndrome elements of 14 factors, and determined the corresponding syndrome-syndrome type relationship. At the same time, items with a load value $>0.4$ were included as candidate items for the syndrome element and symptom relationship model.

\section{Comparison of cluster and factor analyses}

In a comparison of the results of the cluster and factor analyses, the 9 syndrome elements were essentially consistent and were thus retained with reference to clinical practice and expert experience.

\section{Syndrome element and symptom correlation}

Two TCM experts, based on their clinical experience, classified the syndrome elements and determined the correlation between the syndrome elements and symptoms (Table 1). 
Table 2 Screening results of syndrome elements

\begin{tabular}{|c|c|}
\hline Syndrome element & Symptoms or signs \\
\hline Yin deficiency & Dry throat and dry mouth, aversion to heat and sweating or night sweating, tinnitus and deafness, dry eyes \\
\hline Excessive heat & Thirst and desire for cold drinks, constipation and deep-colored urine, polyphagia with tendency to hunger \\
\hline Qi stagnation & Irritability or depression and sighing, distension in the side ribs, distension in the abdomen \\
\hline Damp heat & Heavy limbs, dry mouth and bitter mouth, yellow and greasy tongue coating \\
\hline Blood stasis & $\begin{array}{l}\text { Squamous and dry skin, dark purple lips, localized pain especially at night, dark purple tongue, cyanosis tortuous } \\
\text { and bruising sublingual vein }\end{array}$ \\
\hline
\end{tabular}

\section{Determined elements of each syndrome}

Spearman correlation analysis and binomial logistic regression were used to screen each syndrome element item, and items selected by both statistical methods were determined as the diagnosis items of the syndrome elements.

Spearman correlation analysis was used to analyze the correlation between the various syndrome elements of $\mathrm{DKD}$ and their corresponding items, and the items with a correlation coefficient $\geq 0.4$ with $\mathrm{P}<0.05$ were retained. Stepwise binomial logistic regression analysis was performed for correlation, and items with $P<0.05$ were retained (Table 2).

\section{Scale item assignment}

According to the regression coefficient, the contribution of each syndrome element item was calculated and values assigned to obtain the diagnosis equation of each syndrome element. Y represent the points of each syndrome element. $\mathrm{Y}_{\mathrm{qi}}$ deficiency $=1^{*}$ fatigue $+16^{*} \mathrm{qi}$ deficiency and disinclination to speak $+11^{*}$ lassitude and pain of the loins and knees + $9^{*}$ tinnitus and deafness $+13^{*}$ loose teeth and hair loss; $Y_{\text {blood }}$ deficiency $=11^{*}$ pale and sallow complexion $+8^{*}$ pale lips and fingernails $+15^{*}$ insomnia and dreaminess $+6^{*}$ forgetfulness $+10^{*}$ numbness of hands and feet; $Y_{\text {yin deficiency }}=11^{*}$ dry throat and dry mouth $+13^{*}$ aversion to heat and sweating or night sweating $+11^{*}$ tinnitus and deafness $+15^{*}$ dry eyes; $Y_{\text {yang deficiency }}$ $=10^{*}$ cold limbs +9 *edema of face and feet $+18^{*}$ deficiency of libido $+13^{*}$ frequent urination at night $+13^{*}$ frequent nocturia; $Y_{\text {excessive heat }}=18^{*}$ thirst and desire for cold drinks +
$16^{*}$ polyphagia with tendency to hunger $+16^{*}$ constipation and deep-colored urine; $Y_{\mathrm{qi} \text { stagnation }}=13^{*}$ irritability or depression and sighing $+20^{*}$ distension in the side ribs + $17^{*}$ distension in the abdomen; $Y_{\text {damp heat }}=19^{*}$ heavy limbs $+16^{*}$ dry mouth and bitter mouth $+15^{*}$ yellow and greasy tongue coating; $Y_{\text {blood stasis }}=13{ }^{*}$ squamous and dry skin + $16^{*}$ dark purple lips $+9^{*}$ localized pain especially at night $+12^{*}$ dark purple tongue, cyanosis tortuous and bruising sublingual vein; $Y_{\text {phlegm turbidity }}=16^{*}$ abdominal distension $+18^{*}$ itchy skin $+16^{*}$ sticky mouth.

\section{Diagnosis equation validation and diagnosis threshold determination}

The receiver operating characteristic (ROC) curve was used to validate the diagnosis equation of each syndrome element to obtain the area under the ROC curve (AUC). According to the ROC curve and the principle of maximizing the Youden index, the diagnostic threshold of each syndrome element was determined (Table 3).

\section{DKD diagnostic scale of TCM syndrome elements}

Table 4 shows the DKD diagnostic scale of TCM syndrome elements.

\section{Discussion}

The development of a TCM syndrome scale has 3 main 
Table 3 AUC of each syndrome element and the diagnostic threshold

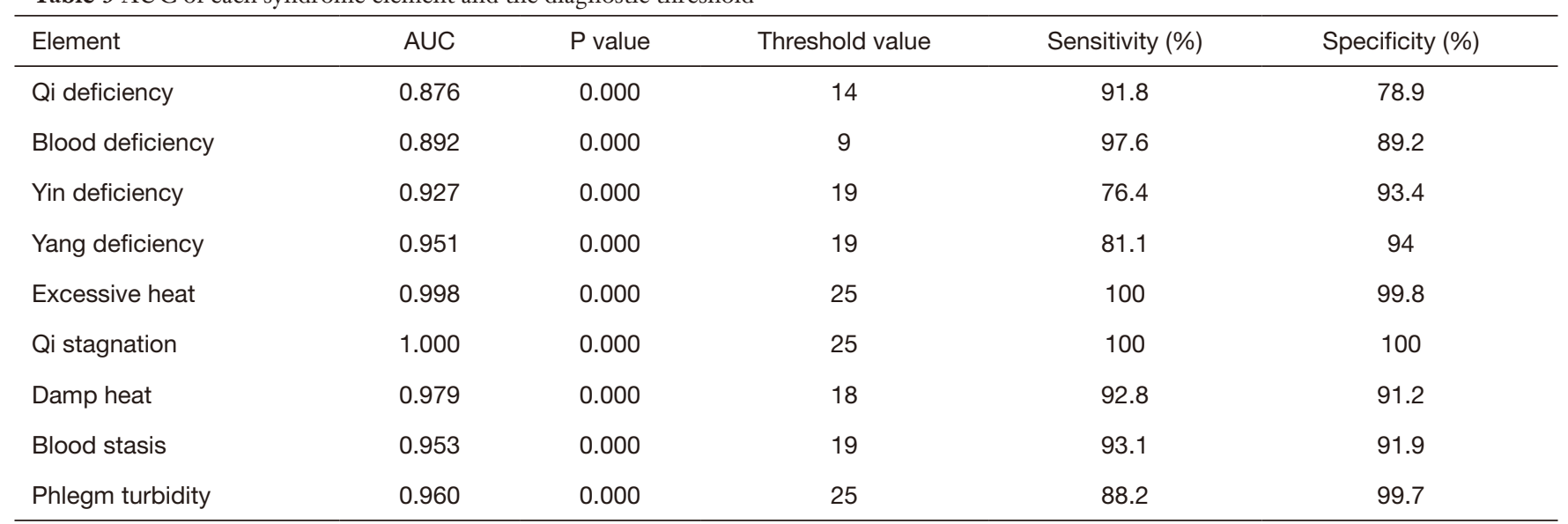

AUC, area under curve.

aspects, namely item screening, item assignment and determination of diagnostic threshold. In this study, frequency, cluster analysis, and factor analysis were used successively to screen and cluster the 4 diagnosis items of TCM; 9 common syndrome elements were extracted, and the syndrome-symptom correlation was determined. Spearman correlation coefficient and binomial logistic regression were used to further screen items for each syndrome element. The 614 -diagnosis items were gradually screened, and 32 valid items were finally retained, which essentially reflected the clinical characteristics of different syndromes of DKD as demonstrated by the clinical validation. In this study, binomial logistic regression was used to unify the total scores of the 9 syndrome elements into 50 points; the items were assigned according to standardized regression coefficients; the ROC curve was used to determine the diagnostic thresholds of different syndrome elements. It is worth mentioning that in the final syndrome scale, certain symptom items had strong diagnostic specificity, such as qi deficiency and disinclination to speak for qi deficiency syndrome; pale and sallow complexion, insomnia and dreaminess for blood deficiency syndrome; heavy limbs for damp heat syndrome. As has been said by Shanghan Lun, "just one symptom can help determine". The findings of our study are applicable to clinical practice. However, attention should be paid to individualized diagnosis and treatment, and patient symptom changes for accurate syndrome differentiation.

Sensitivity and specificity were the main evaluation indicators of the performance of the TCM syndrome scale. The AUC of the 9 syndrome elements included in this scale was between 0.876 and 1. Except for qi deficiency, the AUC of other syndrome elements was $>0.9$. The smallsample clinical validation results showed that the diagnostic sensitivity of each syndrome element was $78.8-100 \%$, the specificity was $84.3-100 \%$, and the accuracy rate was $82.7-100 \%$, which indicated that the scale had good diagnostic performance.

TCM syndrome science aims to make traditional syndromes scientific and standardized in contemporary practice. It can be said that the core and principal body of modern TCM is syndrome science, which is the key point of connection of principle-method-recipemedicines. The starting and end points of principlemethod-recipe-medicines are the application of TCM syndromes in actual cases. With the unremitting efforts of generations of TCM practitioners, the development of TCM syndromes has made considerable progress. However, there are still many problems to be solved in the standardization of TCM syndromes, such as standardization of diseases, symptoms, syndrome names and categories, unification of syndrome classification, and scientific evaluation of clinical manifestations. With the introduction of evidence-based medicine and mathematical statistics, the development of syndrome research has accelerated the process. Researchers (14) have proposed standard quantification of symptoms, signs and syndromes by computational methods based on literature, expert opinion and clinical research. This study of the construction and validation of a DKD diagnostic scale of TCM syndrome elements is consistent with the above proposition. 
Table 4 DKD diagnostic scale of TCM syndrome elements

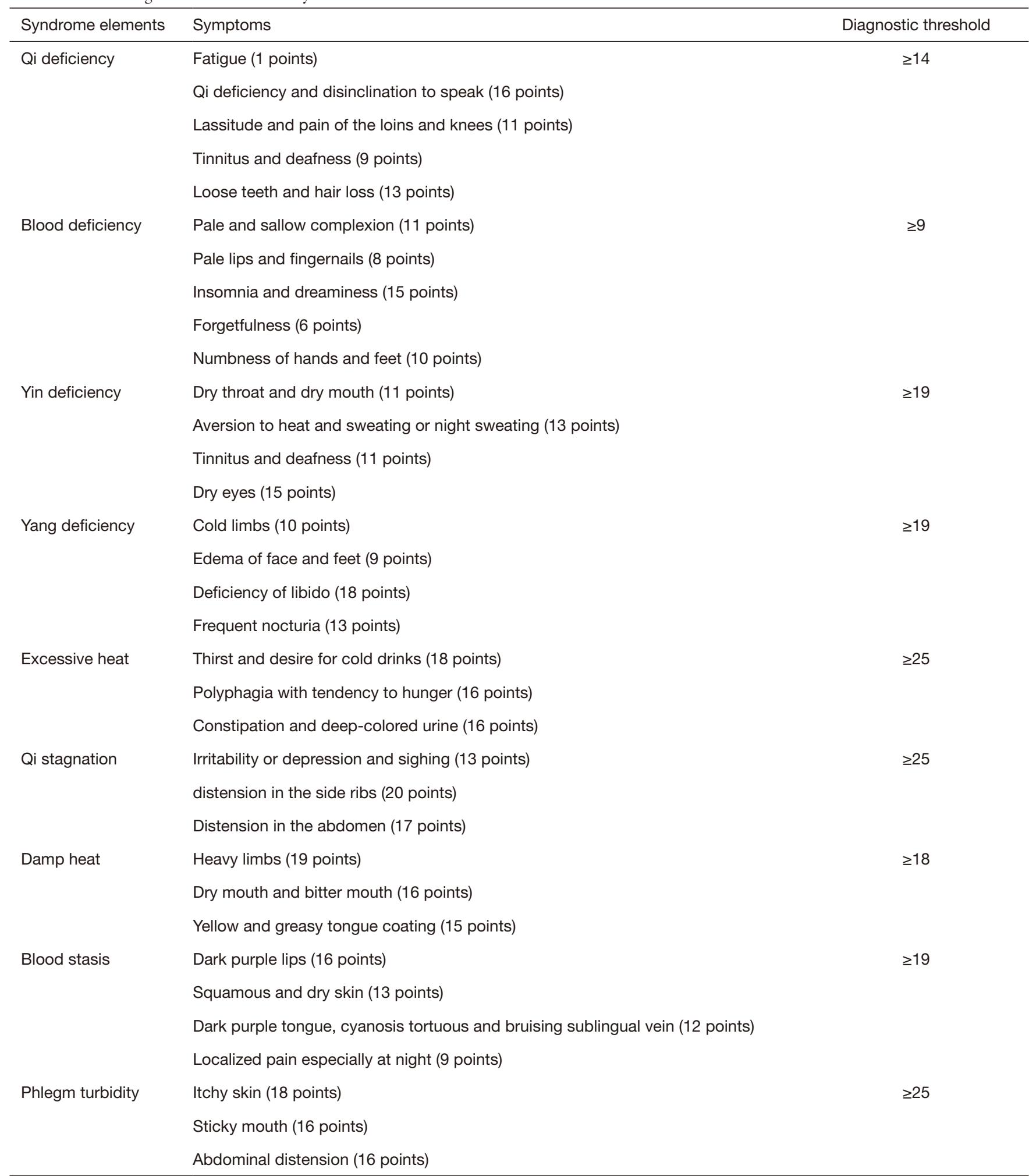

DKD, diabetic kidney disease; TCM, traditional Chinese medicine 
However, there were some limitations to our study. For example, tongue diagnosis and pulse diagnosis are indispensable in TCM diagnosis and treatment, and the current TCM syndrome scale items mostly focus on the content of consultation, and tongue and pulse information are often excluded because of its subjective nature. If objective techniques and methods for tongue diagnosis, pulse diagnosis, and face diagnosis could be introduced into the compilation of TCM scales, it will undoubtedly increase the clinical relevance of the scales.

TCM treatment of DKD is not standardized and mainly based on personal experience, because syndrome differentiation and classification is not unified. The pathogenesis of DKD is excess in nature and deficiency in superficiality, simultaneous occurrence of deficiency and excess syndrome, and the treatment is focus on strengthening vital qi to eliminate pathogenic factor and treating both symptoms and root causes. Above all, DKD scale study is of great clinical value.

Studies provided evidence for the efficacy of TCM from the perspectives of balancing metabolic disorders, inflammatory response, antifibrosis, protecting renal innate cells, and regulating microRNA. TCM may both inhibit or increase the expression of signaling pathways (NF-B, Smad3, PI3K/Akt/mTOR, Smad7 and PTEN) to achieve the goal of DKD treatment (15). DKD could be treated with integrated traditional Chinese and western medicine. On one hand, western medicine treatment could control blood pressure, blood sugar, blood lipids and other indicators, at the same time Chinese medicine treatment based on syndrome differentiation could adjust the balance of yin and yang. Integrated traditional Chinese and Western medicine treatments complement each other in order to slow down the disease progression and improve the quality of life of patients.

The TCM syndrome elements diagnostic scale for DKD is simple and easy for TCM practitioners to perform. The diagnostic scale is valid and reliable for accessing TCM syndrome elements in DKD patients with good diagnostic efficacy. By establishing a TCM syndrome elements diagnostic scale for DKD and determining its cut-off value for diagnosis, we hope to diagnose the TCM syndromes of DKD in a standardized manner.

\section{Acknowledgments}

Funding: This research was supported by the Beijing Municipal Commission of Science and Technology (No.
Z161100001816003, 2016).

\section{Footnote}

Reporting Checklist: The authors have completed the STARD reporting checklist. Available at https://dx.doi. org/10.21037/apm-21-3147

Data Sharing Statement: Available at https://dx.doi. org/10.21037/apm-21-3147

Conflicts of Interest: All authors have completed the ICMJE uniform disclosure form (available at https://dx.doi. org/10.21037/apm-21-3147). The authors have no conflicts of interest to declare.

Ethical Statement: The authors are accountable for all aspects of the work in ensuring that questions related to the accuracy or integrity of any part of the work are appropriately investigated and resolved. All procedures performed in this study involving human participants were in accordance with the Declaration of Helsinki (as revised in 2013). The study was approved by ethics board of Dongzhimen Hospital affiliated to Beijing University of Chinese Medicine (No. ECPJ-BDY-2013-47) and informed consent was taken from all the patients.

Open Access Statement: This is an Open Access article distributed in accordance with the Creative Commons Attribution-NonCommercial-NoDerivs 4.0 International License (CC BY-NC-ND 4.0), which permits the noncommercial replication and distribution of the article with the strict proviso that no changes or edits are made and the original work is properly cited (including links to both the formal publication through the relevant DOI and the license). See: https://creativecommons.org/licenses/by-nc-nd/4.0/.

\section{References}

1. Guo K, Zhang L, Zhao F, et al. Prevalence of chronic kidney disease and associated factors in Chinese individuals with type 2 diabetes: Cross-sectional study. J Diabetes Complications 2016;30:803-10.

2. Zhang L, Long J, Jiang W, et al. Trends in Chronic Kidney Disease in China. N Engl J Med 2016;375:905-6.

3. Bonner R, Albajrami O, Hudspeth J, et al. Diabetic Kidney Disease. Prim Care 2020;47:645-59.

4. GBD Chronic Kidney Disease Collaboration. Global, 
regional, and national burden of chronic kidney disease, 1990-2017: a systematic analysis for the Global Burden of Disease Study 2017. Lancet 2020;395:709-33.

5. Liao T, Zhao K, Huang Q, et al. A randomized controlled clinical trial study protocol of Liuwei Dihuang pills in the adjuvant treatment of diabetic kidney disease. Medicine (Baltimore) 2020;99:e21137.

6. Wang X, Yang H, Zhang L, et al. Network PharmacologyBased Prediction of Mechanism of Shenzhuo Formula for Application to DKD. Evid Based Complement Alternat Med 2021;2021:6623010.

7. Zhang, Yang L, Shergis J, et al. Chinese herbal medicine for diabetic kidney disease: a systematic review and metaanalysis of randomised placebo-controlled trials. BMJ Open 2019;9:e025653.

8. Piao Y, Yin D. Mechanism underlying treatment of diabetic kidney disease using Traditional Chinese Medicine based on theory of Yin and Yang balance. J Tradit Chin Med 2018;38:797-802.

9. Cao X, Wei R, Zhou J, et al. Wenshen Jianpi recipe, a blended traditional Chinese medicine, ameliorates proteinuria and renal injury in a rat model of diabetic nephropathy. BMC Complement Altern Med 2019;19:193.

10. Dong Z, Sun Y, Wei G, et al. A Nucleoside/Nucleobase-
Rich Extract from Cordyceps Sinensis Inhibits the Epithelial-Mesenchymal Transition and Protects against Renal Fibrosis in Diabetic Nephropathy. Molecules 2019;24:4119.

11. Gao X, Shang J, Liu H, et al. A Meta-Analysis of the Clinical Efficacy of TCM Decoctions Made from Formulas in the Liuwei Dihuang Wan Categorized Formulas in Treating Diabetic Nephropathy Proteinuria. Evid Based Complement Alternat Med 2018;2018:2427301.

12. Weng J, Ji L, Jia W, et al. Standards of care for type 2 diabetes in China. Diabetes Metab Res Rev 2016;32:442-58.

13. Zhang XH. Characteristics of TCM syndromes and physical and chemical indexes in the occurrence and development of diabetic kidney disease. Beijing: Beijing University of Chinese Medicine, 2015.

14. Li JS, Yu XQ, Hu JL. Insights and approaches of establishing standardization of TCM syndrome. Journal of Henan University of Traditional Chinese Medicine 2004;19:4-6.

15. Lu Z, Zhong Y, Liu W, et al. The Efficacy and Mechanism of Chinese Herbal Medicine on Diabetic Kidney Disease. J Diabetes Res 2019;2019:2697672.

(English Language Editor: K. Brown)

Cite this article as: Jiang $M$, Lou XE, Zhang X, Nan Q, Gao X, Liu H. Development and validation of the diagnostic scale of traditional Chinese medicine syndrome elements for diabetic kidney disease. Ann Palliat Med 2021;10(12):12291-12299. doi: 10.21037/apm-21-3147 